\title{
On the Expression of Graph Properties in some Fragments of Monadic Second-Order Logic
}

\author{
Bruno Courcelle
}

\begin{abstract}
We review the expressibility of some basic graph properties in certain fragments of Monadic Second-Order logic, like the set of Monadic-NP formulas. We focus on cases where a property and its negation are both expressible in the same (or in closely related) fragments. We examine cases where edge quantifications can be eliminated and cases where they cannot. We compare two logical expressions of planarity: one of them is constructive in the sense that it defines a planar embedding of the considered graph if it is planar and 3-connected, and the other, logically simpler, uses the forbidden Kuratowski subgraphs.
\end{abstract}

\section{Introduction}

By considering graphs as logical structures one can express their properties by logical formulas. From the logical expression of a graph property, one can obtain upper bounds to its complexity, over all graphs or over specific classes of graphs: see for instance [2, $4,10,12,14,18,25,26]$. One can also specify by logical formulas certain constructions like the unique plane embedding of a planar 3-connected graph.

We will use Monadic Second-Order logic (MS logic) to define these properties and constructions. This language is of special interest for the following reasons: it is powerful enough to express non-first-order graph properties like connectivity, acyclicity, planarity, $k$-colorability for each fixed $k$ and is nevertheless algorithmically tractable: every MS (Monadic Second-Order) property is testable in linear time on "tree-structured" graphs, a notion which has of course a precise definition ([2, 4, 10]). MS logic is also closely connected with finite-state automata: for finite binary trees, recognizability by finite-state automata is equivalent to definability by an MS formula. This connection yields effective constructions of linear-time verification algorithms. It extends also to infinite trees and culminates with Rabin's theorem which is fundamental in semantics (Emerson [11]).

Second-order logic is of course more powerful than MS logic. However, it is in a sense too powerful: the connection with automata does not extend and no general efficient verification method is known, even for tree-structured graphs. However, it is interesting because of its connections with complexity classes like NP and the polynomial hierarchy

1991 Mathematical Subject Classification. Primary 03C13; Secondary 05C10, 05C15.

Work supported by ESPRIT Basic Research project: "COMPUGRAPH 2". 


\section{BRUNO COURCELLE}

$[12,18,26]$. We will discuss some fragements of MS logic (like the class of monadicNP formulas) which have been introduced in the hope of proving that NP $\neq$ coNP by logical techniques $([1,3,12,13,15,18])$.

This survey is organized a follows. Section 1 reviews MS logic and some of its fragments of special interest in the field of descriptive complexity. We consider basic graph properties like connectivity or the existence of disjoint paths between given vertices, and we compare the logical expression of a property with that of its negation. In Section 2 we show that the orientations of an undirected graph can be specified by MS formulas using quantifications on sets of edges, and that they cannot be (in general) with formulas using vertex set quantifications only.

In Section 3 we show that edge set quantifications can be eliminated in MS formulas expressing properties of planar graphs or of graphs of bounded degree or tree-width. In Section 4 we consider chordal graphs and planar graphs. We observe that both classes can be defined "negatively" (i.e., by forbidden configurations) in a simpler way than "constructively". We establish the new result that the unique planar embedding of a 3connected planar graph is definable by MS formulas.

Section 5 reviews further topics of interest in this field and presents some open problems.

\section{Monadic second-order logic}

A graph can be defined as a relational structure $G=\left\langle\mathbf{V}_{G}\right.$, $\left.\mathbf{e d g}_{G}\right\rangle$ where $\mathbf{V}_{G}$, the set of vertices of the graph, is the domain of the structure and $\operatorname{edg}_{G}$ is a binary relation on $\mathbf{V}_{G}$ representing the edges. Hence, a closed logical formula written with the binary relation symbol edg expresses a graph property in a natural way.

One can thus classify graph properties according to the logical complexity of the formulas expressing them. This field is called descriptive complexity. We introduce or recall the definitions of a few languages.

We will denote by $F O, M S$ and $S O$ the sets of first-order, monadic second-order and second-order formulas respectively. We will denote by $\exists_{\text {set }} F O$ the set of formulas of the special form $\exists X_{1}, \ldots, X_{k} \varphi$ where $k \geq 0, X_{1}, \ldots, X_{k}$ are set variables and $\varphi$ is $F O$ (the set variables $X_{1}, \ldots, X_{k}$ can occur in $\varphi$ in atomic formulas of the form $x \in X_{i}$ where $x$ is an individual variable); these formulas are termed monadic $-\Sigma_{1}^{1}$ in [1] and monadic$N P$ in $[3,15]$.

We will denote by $F O^{T C}$ the set of first-order formulas constructed with special atomic formulas representing transitive closures of binary relations defined by existential first-order formulas; these atomic formulas will be written $\operatorname{TC}_{x, y}(\varphi)(u, v)$ where $\varphi$ is an existential first-order formula that can have other free variables than $x$ and $y$, say $z_{1}, \ldots, z_{k}, X_{1}, \ldots, X_{n}$. We will define the meaning of these new formulas. If $R$ is a binary relation, we will denote by $R^{+}$its transitive closure. Furthermore, we will denote by the same symbol a variable and a value assigned to it. Here is the definition.

For every assignment of values to $z_{1}, \ldots, z_{k}, X_{1}, \ldots, X_{n}$, for every $u, v$ in the domain $\mathbf{D}_{S}$ of the considered structure $S$, then

$$
\begin{gathered}
\mathrm{TC}_{x, y}(\varphi)(u, v) \text { holds iff }(u, v) \in R^{+} \text {where } R \subseteq \mathbf{D}_{S} \times \mathbf{D}_{S} \text { is defined by : } \\
R=\left\{(x, y) \in \mathbf{D}_{S} \times \mathbf{D}_{S} / S \vDash \varphi\left(x, y, z_{1}, \ldots, z_{k}, X_{1}, \ldots, X_{n}\right)\right\} .
\end{gathered}
$$

Note that this transitive closure constructor is used for binary relations over domains of structures (and not over $n$-fold products of domains of structures), which, furthermore, are defined by existential first-order formulas (and not by arbitrary first-order formulas). 
MONADIC SECOND-ORDER LOGIC

By replacing $F O$ by $F O^{T C}$ in the definition of $\exists_{\text {set }} F O$, we obtain the set of formulas $\exists_{\text {set }} F O^{T C}$. Since transitive closures are definable by $M S$ formulas we have

$$
F O \subset \exists_{\mathrm{set}} F O \subset \exists_{\mathrm{set}} F O^{T C} \subset M S .
$$

Examples will prove that the corresponding hierarchy of graph properties is strict. Since formulas in $F O^{T C}$ denote polynomial (and even $N L$ ) properties [18], formulas in $\exists_{\text {set }} F O^{T C}$ denote $N P$ properties. The second-order formulas denote the properties in the polynomial hierarchy. It is proved in Makowsky and Pnueli [21] that MS properties can be arbitrarily high in the polynomial hierarchy.

Defining graphs as we did has two drawbacks: first, one defines simple graphs only, (simple means that there are no multiple edges) and second, quantified variables cannot denote edges or sets of edges. Even for expressing properties of simple graphs, quantification on sets of edges is essential in certain cases as we will see.

For every graph $G$ we let $|G|_{1}$ be the above defined structure $\left\langle\mathbf{V}_{G}, \mathbf{e d g}_{G}\right\rangle$. A simple graph $G$ is completely determined up to isomorphism by the structure $|G|_{1}$. For an arbitrary graph $G$ we let $|G|_{2}:=\left\langle\mathbf{V}_{G} \cup \mathbf{E}_{G}\right.$, inc $\left.\mathbf{i}_{G}\right\rangle$ where $\mathbf{E}_{G}$ is the set of edges and inc $G$ is the ternary relation such that inc $G(x, y, z)$ holds iff $x$ is an edge from $y$ to $z$. (If $G$ is undirected $\operatorname{inc}_{G}(x, y, z)$ holds iff $\operatorname{inc}_{G}(x, z, y)$ holds). An element $x$ of $\mathbf{V}_{G} \cup \mathbf{E}_{G}$ is an edge iff there exist $y, z$ such that $\operatorname{inc}_{G}(x, y, z)$ holds. Every graph $G$ is completely determined, up to isomorphism, by the structure $|G|_{2}$.

For each of the sets of formulas $F O, M S, \exists_{\text {set }} F O$ etc... the subscript 1 (resp. 2) will indicate that the formulas are written with the relation symbol edg (respectively inc) hence that they express properties of graphs $G$ represented by the structures $|G|_{1}$ (respectively $|G|_{2}$ ). We will see below that non-planarity is expressed by a formula in $\exists_{\text {set }} F O_{1}$ ( i.e., a monadic-NP formula in the terminology of [15]). We will see that directed reachability is expressed by a formula in $\exists_{\text {set }} F O_{2}$ whereas (by [1]) it is not expressible by any formula in $\exists_{\mathrm{set}} F O_{1}$.

We will now review some basic graph properties that are significant for the comparison of the expressive powers of the various fragments of MS logic we have introduced. We shall only consider finite graphs (except in a short discussion in Section 5). In order to shorten statements and without any significant loss of generality, we will use the following

Convention: The term graph will mean finite simple loop-free graph.

\section{Vertex colorability}

For each $k$, (vertex) $\boldsymbol{k}$-colorability is $\exists_{\text {set }} F O_{1}$ (i.e., is expressible by a formula in $\exists_{\text {set }} F O$ relative to the representation of a graph $G$ by the structure $|G|_{1}$ ). The formula is easy to construct. For each $k \geq 3$ this property in $N P$-complete. Furthermore $N P=$ coNP iff Non-3-colorability is expressible by a second-order formula of the form $\exists R_{1}, \ldots, R_{n} \varphi$ where $R_{1}, \ldots, R_{n}$ are relation variables (possibly not unary) and $\varphi$ is first-order. (See [1]). Cosmadakis has proved in [3] that Non-3-colorability is not a $\exists_{\text {set }} F O_{1}$ property. 
BRUNO COURCELLE

Connectivity properties

If $R \sqsubseteq D \times D$ and $X \subseteq D$, we say that $X$ is $R$-closed if for all $x, y \in D$, if $x$ $\in X$ and $(x, y) \in R$ then $y \in X$. (This is clearly a first-order property). Nonconnectivity is $\exists_{\text {set }} F O_{1}$ since it can be expressed by the formula

$$
\exists X\left(\exists x(x \in X) \wedge \exists y(\neg y \in X) \wedge " X \text { is (edg } \cup \mathbf{e d g}^{-1}\right) \text {-closed"). }
$$

(The text between quotes defines the meaning of the subformula it stands for; this formula is straightforward to write. Text between quotes will be used in this way in the sequel in order to make formulas readable.)

Since Connectivity is not $\mathrm{FO}_{1}$ (Gaifman [16]), this proves that formulas in $\exists$ set $F O$ are strictly more expressive than first-order ones. Similarly, DirectedUnreachability $(x, y)$ (the non-existence of a directed path from $x$ to $y$ ) is expressed by the following formula in $\exists_{\text {set }} F O_{1}$ :

$$
\exists X(x \in X \wedge \neg y \in X \wedge " X \text { is edg-closed"). }
$$

In undirected graphs, edg denotes a symmetric relation and formula (2) expresses Unreachability $(x, y)$, i.e., the non-existence of a path between $x$ and $y$. (Any two vertices in a path are distinct except possibly the two ends in the case of a cycle; in a directed graph $G$ we distinguish a path, where edges can be traversed in either direction, from a directed path, where they are all traversed in the natural direction.)

DirectedReachability $(x, y)$ (i.e., the existence of a directed path from $x$ to $y$ ) is expressed by the formula $\mathrm{TC}_{u, v}(\operatorname{edg}(u, v))(x, y)$ and by a formula in $\exists_{\text {set }} F O_{2}$ that we now construct. We let first QuasiPath $(X, x, y)$ be the first-order formula expressing the following :

$X$ is a set of edges, $x$ and $y$ are vertices, $x \neq y, x$ is the origin of a unique edge in $X$ and the target of none, $y$ is the target of a unique edge in $X$ and the origin of none, every vertex other than $x$ and $y$ that is incident to an edge of $X$ is the origin of a unique edge in $X$ and the target of a unique edge in $X$.

Since graphs are finite, these conditions express that $X$ is the set of edges of a directed path from $x$ to $y$ augmented possibly with the vertices of pairwise disjoint cycles that are disjoint from the path. Hence DirectedReachability $(x, y)$ is equivalent to $\exists X$ QuasiPath $(X, x, y)$ and is thus a $\exists_{\mathrm{set}} F O_{2}$ property. Ajtai and Fagin [1] proved that no formula in $\exists_{\mathrm{set}} F O_{1}$ can express this property.

The situation is simpler for $\operatorname{Reachability}(x, y)$ (in undirected graphs or in directed graphs but for nonnecessarily directed paths). This property can be expressed by the $\exists_{\text {set }} F O_{1}$ formula $\exists X$ QuasiPath' $(X, x, y)$ where QuasiPath' $(X, x, y)$ is the first-order formula :

$x \in X \wedge y \in X \wedge x \neq y \wedge$ "each of $x$ and $y$ is adjacent to a unique element of $X " \wedge$ "every vertex in $X-\{x, y\}$ is adjacent to exactly two vertices of $X ")$.

(This construction, due to $\mathrm{P}$. Kannellakis, is given in [1]; one can use the same idea to construct a formula in $\exists_{\text {set }} F O_{1}$ expressing DirectedReachability $(x, y)$ in directed acyclic graphs). 
MONADIC SECOND-ORDER LOGIC

Connectivity is expressed by : $\exists x \forall y[x=y \vee \operatorname{Reachability}(x, y)]$ hence by a formula in $F O_{1}^{T C}$. It is known from Fagin [13] that Connectivity is not $\exists_{\mathrm{set}} F O_{1}$. (It is not $\exists_{\text {set }} F O_{2}$ either since the proof of [13] uses graphs of degree at most 2, and since formulas in $\exists_{\text {set }} F O_{1}$ and in $\exists_{\text {set }} F O_{2}$ express the same properties of these graphs by Theorem (3.3) below.)

Disjoint paths in undirected graphs

Let $k \geq 2$. k-DisjointPaths $\left(x_{1}, \ldots, x_{k} ; y_{1}, \ldots, y_{k}\right)$ is the property saying that: $x_{1} \neq y_{1}, \ldots, x_{k} \neq y_{k}$ and there exist $k$ paths $p_{1}, \ldots, p_{k}$ linking $x_{1}$ to $y_{1}, \ldots$, $x_{k}$ to $y_{k}$ respectively, such that every vertex common to two paths $p_{i}$ and $p_{j}, j \neq i$, belongs to $\left\{x_{i}, y_{i}\right\} \cap\left\{x_{j}, y_{j}\right\}$.

From the construction done for Reachability it is not hard to see that $\mathbf{k}$ DisjointPaths $\left(x_{1}, \ldots, x_{k} ; y_{1}, \ldots, y_{k}\right)$ is expressible by a formula in $\exists_{\text {set }} F O_{1}$ with $k$ existential set quantifications (representing the $k$ desired paths).

k-Separability $(x, y)$ is the property saying that $x$ and $y$ are distinct non-adjacent vertices and that there exists a set $A$ of at most $k$ vertices such that every path from $x$ to $y$ goes through $A$. It is $\exists_{\text {set }} F O_{1}$ since it can be formulated as follows:

$\exists X, Y[" x \in X-(X \cap Y) " \wedge " y \in Y-(X \cap Y) " \wedge$ "every vertex belongs to $X \cup Y " \wedge " X \cap Y$ has cardinality $\leq k " \wedge$ " there is no edge between a vertex of $X-(X \cap Y)$ and a vertex of $Y$ - $(X \cap Y)$ "].

The negation of a $\exists_{\mathrm{set}} F O_{1}$ property is usually not a $\exists_{\mathrm{set}} F O_{1}$ one. However, it follows from Menger's theorem (see Tutte [28]) that the negation of $\mathbf{k}$ $\operatorname{Separability}(x, y)$ is equivalent to

$$
x=y \vee \operatorname{edg}(x, y) \vee(\mathbf{k}+\mathbf{1}) \text {-DisjointPaths }(x, x \ldots, x ; y, y \ldots, y)
$$

hence is also $\exists_{\mathrm{set}} F O_{1}$. We do not know whether the negation of $\mathbf{k}$ DisjointPaths $\left(x_{1}, \ldots, x_{k} ; y_{1}, \ldots, y_{k}\right)$ is $\exists_{\text {set }} F O_{1}$.

Forbidden configurations and nonplanar graphs

Let $H$ and $G$ be undirected graphs. We say that $H$ embeds in $G$ iff there is a subgraph $G^{\prime}$ of $G$ which is isomorphic to a graph obtained from $H$ by the replacement of some edges by disjoint paths. Hence $G$ is nonplanar iff either $K_{5}$ or $K_{3,3}$ embeds in $G$. (For $n \geq 2, K_{n}$ is the complete undirected graph with $n$ vertices and $K_{n, p}$ is the graph with vertices $-n, \ldots,-1,1, \ldots, p$ and undirected edges between $-i$ and $j$ for positive $i, j$.)

For every fixed undirected graph $H$ without isolated vertices the property of an arbitrary graph $G$ that $H$ embeds in $G$ is expressible by a formula in $\exists_{\text {set }} F O_{1}$ constructed as follows. We let $\mathbf{V}_{H}=\{1, \ldots, n\}, \mathbf{E}_{H}=\left\{\left(i_{1}, j_{1}\right), \ldots,\left(i_{k}, j_{k}\right)\right\}$ for some $i_{1}, \ldots, i_{k}, j_{1}, \ldots, j_{k} \in\{1, \ldots, n\}$. Then $H$ embeds in $G$ iff $|G|_{1}$ satisfies the formula :

$$
\exists x_{1}, \ldots, x_{k}, y_{1}, \ldots, y_{k}, z_{1}, \ldots, z_{n}
$$

$$
\text { [k-DisjointPaths } \left.\left(x_{1}, \ldots, x_{k} ; y_{1}, \ldots, y_{k}\right) \wedge \wedge{ }_{1 \leq i \leq n} E_{i}\right]
$$




\section{BRUNO COURCELLE}

where for each $i, E_{i}$ is the conjunction of the equalities $x_{\ell}=z_{i}, y_{m}=z_{i}$ such that $i_{\ell}=j_{m}=i$ and of the inequalities $x_{\ell} \neq z_{i}, y_{m} \neq z_{i}$ such that $i_{\ell} \neq i, j_{m} \neq i$. It is a routine task to rewrite this formula into one in $\exists_{\mathrm{set}} F O_{1}$. It follows that NonPlanarity is a $\exists_{\text {set }} F O_{1}$ property. We will present in Section 4 a characterization of Planarity (for 3-connected graphs) by a formula in $\exists_{\mathrm{set}} F O_{1}^{T C}$ which specifies a planar embedding.

Trees

We consider undirected graphs. Such a graph $G$ has a cycle iff there are adjacent vertices $x$ and $y$ connected by a path not using the edge linking them. This can be expressed by the following formula in $F O_{1}^{T C}$ :

$$
\exists x, y\left[\operatorname{edg}(x, y) \wedge \mathrm{TC}_{u, v}(\operatorname{edg}(u, v) \wedge \neg\{x=u \wedge y=v\} \wedge \neg\{x=v \wedge y=u\})(x, y)\right] .
$$

It follows that Tree (the property of being a tree) is expressible for undirected graphs by a formula in $F O O_{1}^{T C}$. An undirected graph is not a tree iff it is not connected or if $K_{3}$ embeds in it. Hence the negation of the property Tree is $\exists_{\text {set }} F O_{1}$.

Monadic Second-Order logic on paths and trees

Let $\mathbb{P}$ be the class of directed paths. For every formula $\varphi$ in $M S_{1}$ with free variables $X_{1}, \ldots, X_{k}$ one can construct a formula $\psi$ in $\exists_{\text {set }} F O_{1}$ with the same free variables that is equivalent to $\varphi$ on all directed paths. We mean by equivalent that for every $G \in \mathbb{P}$, for every $X_{1}, \ldots, X_{k} \subseteq \mathbf{V}_{G}$ we have :

$$
|G|_{1} \vDash \psi\left(X_{1}, \ldots, X_{k}\right) \quad \text { iff } \quad|G|_{1} \vDash \varphi\left(X_{1}, \ldots, X_{k}\right)
$$

Every tuple $\left(G, X_{1}, \ldots, X_{k}\right)$ where $G \in \mathbb{P}$ and $X_{i} \subseteq \mathbf{V}_{G}$ can be made as follows into a word $w\left(G, X_{1}, \ldots, X_{k}\right)$ in $A^{+}$(the set of nonempty words over $A$ ) where $A=\{0,1\}^{k}:$ if $G$ is the graph $v_{1} \rightarrow v_{2} \rightarrow \ldots \rightarrow v_{n}$ then $w\left(G, X_{1}, \ldots, X_{k}\right)$ is the word $a_{1} a_{2} \ldots a_{n}$ where $a_{i}$ is the sequence of bits $b_{1} \ldots b_{k}$ such that $b_{j}=1$ iff $v_{i} \in$ $\left.X_{j}\right)$. Then $\left\{w\left(G, X_{1}, \ldots, X_{k}\right) / G \in \mathbb{P},|G|_{1} \vDash \varphi\left(X_{1}, \ldots, X_{k}\right)\right\}$ is a regular language, and from a finite-automaton with $m$ states recognizing it, one obtains a formula $\psi$ of the desired form with $m$ existential set quantifiers. (However, the number $m$ is a tower of exponentials in the alternation depth of $\varphi$.) See Thomas [27, Thm 3.2]. Hence monadic second-order logic is equivalent on $\mathbb{P}$ to its fragment $\exists_{\text {set }} F O_{1}$. Similar results hold for trees of degree $\leq k$ for any fixed $k$ (see [27, Thm 11.1]) and for trees of unbounded degree [29].

(1.1) Open problem : On which other classes of graphs is monadic second-order logic equivalent to its fragment $\exists_{\mathrm{set}} F O_{1}$ or to a related one?

\section{Summary}

We summarize in a table the facts we have discussed (or will discuss) concerning the expressibility of some graph properties, and of their negation. Except for DirectedReachability, all graphs are undirected. 
MONADIC SECOND-ORDER LOGIC

\begin{tabular}{|c|c|c|}
\hline Property & itself & its negation \\
\hline 3-Colorability & $\exists_{\mathrm{set}} F O_{1}$ & not $\exists_{\mathrm{set}} F O_{1}$ \\
\hline Connectivity & $F O_{1}^{T C}$, not $\exists_{\text {set }} F O_{2}$ & $\exists_{\text {set }} F O_{1}$ \\
\hline Reachability $(x, y)$ & $\exists_{\text {set }} F O_{1}$ & $\exists_{\mathrm{set}} F O_{1}$ \\
\hline D $^{\text {ted }}$ Reachability $(x, y)$ & $\begin{array}{c}F O_{1}^{T C}, \exists_{\mathrm{set}} F O_{2} \\
\text { not } \exists_{\mathrm{set}} F O_{1}\end{array}$ & $\exists_{\text {set }} F O_{1}$ \\
\hline k-Separability $(x, y)$ & $\exists_{\mathrm{set}} F O_{1}$ & $\exists_{\text {set }} F O_{1}$ \\
\hline k-DisjointPaths(...) & $\exists_{\mathrm{set}} F O_{1}$ & \\
\hline Planarity & $\exists_{\mathrm{set}} F O{ }_{1}^{T C}$ & $\exists_{\text {set }} F O_{1}$ \\
\hline Tree & $F O_{1}^{T C}$ & $\exists_{\mathrm{set}} F O_{1}$ \\
\hline Hamiltonicity & $\exists_{\mathrm{set}} F O_{2}^{T C}$, not $M S_{1}$ & \\
\hline Chordality & $\exists_{\mathrm{set}} F O_{2}^{T C}$ & $\exists_{\text {set }} F O_{1}$ \\
\hline $\begin{array}{l}\text { Any } M S \text {-property on } \\
\text { words or trees }\end{array}$ & $\exists_{\text {set }} F O_{1}$ & $\exists_{\text {set }} F O_{1}$ \\
\hline
\end{tabular}

The blank boxes in the negation column mean that we know nothing better than the straightforward negation of the formula expressing the property. Hamiltonicity will be discussed in Section 2 and 3. Planarity and chordality will be discussed in Section 4.

\section{The definition of orientations by $\mathrm{MS}_{2}$ formulas}

For a directed graph $G$, we denote by und $(G)$ the underlying undirected graph obtained by forgetting the orientations of edges; two parallel but opposite edges of $G$ get fused into a unique edge in und $(G)$.

Let us assume we know how to express by an $M S_{i}$ formula a property $P$ of directed graphs. Can one express by an $M S_{i}$ formula the property $P^{\mathbf{u n d}}$ of undirected graphs defined by :

$$
P^{\left.\mathbf{u n d}_{(}\right)}: \Leftrightarrow P\left(G^{\prime}\right) \text { holds for some directed graph } G^{\prime} \text { such that } G=\operatorname{und}\left(G^{\prime}\right) \text { ? }
$$

The answer is "yes" if $i=2$ and "not always" if $i=1$. The reason is that the orientations of an undirected graph can be defined by $M S_{2}$ formulas but not by $M S_{1}$ ones. We first show the counterexample and then give the construction.

(2.1) Proposition ([7]): The property that an undirected graph has a Hamiltonian cycle is not $\mathrm{MS}_{1}$ but is of the form $\mathrm{P}^{\mathrm{und}}$ for some $\mathrm{MS}_{1}$ property $\mathrm{P}$ of directed graphs .

Proof : Note that by our initial convention, Hamiltonian graphs have at least 3 vertices. We let $P(H)$ be the following property of a directed graph $H$ :

there are at least 3 vertices, the reflexive and transitive closure of the relation $\mathbf{e d g}_{H}$ is a linear order on $\mathbf{V}_{H}$ and there is an edge from the first to the last element of $\mathbf{V}_{H}$ (where first and last are relative to this order). 


\section{BRUNO COURCELLE}

This property is actually in $F O O_{1}^{T C}$. It is not hard to see that Hamiltonicity is equivalent to $P$ und .

Assume by contradiction that Hamiltonicity is expressed by an $M S_{1}$ formula $\varphi$. Observe that $K_{n, m}$ is Hamiltonian iff $n=m$. Consider now a word $w$ in $\{a, b\}^{+}$of length at least 3 , which is represented by a structure $\|w\|$ having a domain which is the set of occurrences of letters and a predicate $\mathbf{p}_{a}(x)$ which holds iff $x$ is an occurrence of letter $a$. By letting $\operatorname{edg}(x, y): \Leftrightarrow\left(\mathbf{p}_{a}(x) \wedge \neg \mathbf{p}_{a}(y)\right) \vee\left(\mathbf{p}_{a}(y) \wedge \neg \mathbf{p}_{a}(x)\right)$, one defines in $\|w\|$ the structure $\left|K_{n, m}\right|_{1}$ where $m$ (resp. $n$ ) is the number of occurrences of $a$ (resp. of $b$ ). The existence of $\varphi$ implies the existence of a monadic second-order formula $\psi$ such that $\|w\| \vDash \psi$ iff $w$ has as many $a^{\prime} s$ and $b^{\prime} s$. This is not possible because the language defined in this way by $\psi$ is not regular (whereas every $M S$ definable language is regular ([27, thm 3.2]). Hence Hamiltonicity is not $M S_{1}$-definable. (This argument is from Makowsky [20]).

We now consider the definition of orientations by $M S_{2}$ formulas. In order to define an orientation of an undirected graph $G$ assumed to be connected, we choose a set of edges $X$ and a vertex $u$ such that $X$ is the set of edges of a depth-first search (dfs) spanning tree of $G$ with root $u$. Call this tree $T$. For vertices $z, y$ we let $z<_{T} y$ if $z$ $\neq y$ and $z$ is on the (unique) path in $T$ from the root to $y$. This tree is depth-first search iff the two ends of every edge of the graph are comparable under $<_{T}$.

We let $G(X, u)$ be the directed graph obtained from $G$ by directing from $z$ to $y$ an edge with ends $y$ and $z$ such that $z<T y$.

Let now $Y$ be an arbitrary subset of $\mathbf{E}_{G}$. We define $G(X, u, Y)$ by directing the edges of $\mathbf{E}_{G}-Y$ as in $G(X, u)$ and those of $Y$ in the opposite direction. It is clear that every directed graph $H$ such that $\operatorname{und}(H)=G$ is of the form $G(X, u, Y)$ for some $X, u$ and $Y$. (One can even choose $X, u$ arbitrarily so as they define a dfs tree of $G$ and then choose $Y$.)

We describe more formally the general construction which works for all, non necessarly connected, graphs. We let $\varphi(X, U, Y)$ be the $F O_{2}^{T C}$ formula expressing the following properties of $X, U, Y$ in a given graph $G$ (where we define an $X$-path as a path all edges of which are in a given set $X$ ):

(1) $U$ is a set of vertices such that every vertex of $G$ is linked by some path to one and only one vertex of $U$;

(2) $X$ is a set of edges and the subgraph of $G$ with set of vertices $\mathbf{V}_{G}$ and set of edges $X$ has no cycle and any two vertices of $G$ linked by a path are linked by an $X$ path;

(3) the two ends of every edge of $G$ belong to an $X$-path one end of which is in $U$;

(4) $Y \subseteq \mathbf{E}_{G}$.

We let also $\psi(X, U, Y, e, x, y)$ be the $F O_{2}^{T C}$ formula such that, whenever $\varphi(X$, $U, Y$ ) holds, we have the following for all $e \in \mathbf{E}_{G}, x, y \in \mathbf{V}_{G}$ :

(5) if $\psi(X, U, Y, e, x, y)$ holds then $x$ and $y$ are the two ends of $e$ and either $e$ is in $Y$ and $y$ is on the (unique) $X$-path from $u$ to $x$ where $u$ is the unique vertex in $U$ linked by some path to $y$,

or $e$ is not in $Y$ and $x$ is on the $X$-path from $u$ to $y$ where $u$ is as above. 
MONADIC SECOND-ORDER LOGIC

Having fixed $X, U, Y$ satisfying (1)-(3), the inc $H$ relation of $H=G(X, U, Y)$ is now defined as follows :

$$
\operatorname{inc}_{H}(e, x, y): \Leftrightarrow \operatorname{inc}_{G}(e, x, y) \wedge[x=y \vee \psi(X, U, Y, e, x, y)]
$$

(2.2) Proposition ([7]): Let $\mathrm{G}$ be undirected. For all sets $\mathrm{X}, \mathrm{U}$, Y such that $|\mathrm{G}|_{2}$ $\vDash \varphi(\mathrm{X}, \mathrm{U}, \mathrm{Y})$ the graph $\mathrm{H}=\mathrm{G}(\mathrm{X}, \mathrm{U}, \mathrm{Y})$ is an orientation of $\mathrm{G}$, and the ternary relation inc is $_{\mathrm{H}}$ is definable in $|\mathrm{G}|_{2}$ by an $\mathrm{FO}_{2}^{\mathrm{TC}}$ formula in terms of $\mathrm{X}, \mathrm{U}, \mathrm{Y}$. For all sets $\mathrm{X}, \mathrm{U}$ such that $|\mathrm{G}|_{2} \vDash \varphi(\mathrm{X}, \mathrm{U}, \varnothing)$, for every orientation $\mathrm{H}$ of $\mathrm{G}$ one can find $\mathrm{Y}$ such that $\mathrm{H}=\mathrm{G}(\mathrm{X}, \mathrm{U}, \mathrm{Y})$.

(2.3) Corollary ([7]): For every $\mathrm{MS}_{2}$ property $\mathrm{P}$ of directed graphs, the property Pund is also $\mathrm{MS}_{2}$.

Proof : Let $P$ be expressed by $\theta$ in $M S_{2}$. Then $P^{\text {und }}$ is expressed by the formula $\exists X, U, Y\left[\varphi(X, U, Y) \wedge \theta^{\prime}\right]$ where $\theta^{\prime}$ is obtained from $\theta$ by replacing every atomic formula inc $(e, x, y)$ by the formula inc $(e, x, y) \wedge \psi(X, U, Y, e, x, y)$ (after the necessary renamings of bound variables have been done as usual).

It follows from Proposition (2.1) that no similar construction giving Proposition (2.2) can be done with $M S_{1}$ formulas. However, for restricted types of graphs we will see in the next section that orientations can be defined by $M S_{1}$ formulas.

\section{The elimination of edge quantifications}

Let us recall that all graphs are simple. It is not hard to see that quantifications on edges are not necessary in first-order formulas. Roughly speaking one can replace $\exists e[" e$ is an edge" $\wedge \varphi$ ] by $\exists x, y\left[" x\right.$ and $y$ are vertices forming an edge" $\left.\wedge \varphi^{\prime}\right]$ where $\varphi^{\prime}$ is an adequate translation of $\varphi$. A similar result holds in second-order logic because an $n$-ary relation on $\mathbf{E}_{G}$ can be handled as a $2 n$-ary relation on $\mathbf{V}_{G}$. However this technique does not work for monadic second-order logic because a set of edges is a binary relation on vertices, and not a unary one. Actually, a quantification on sets of edges can be seen as a restricted form of quantification on binary relations on vertices.

Is $\mathrm{MS}_{2}$ more expressive than $\mathrm{MS}_{1}$ ?

We have seen that DirectedReachability is $\exists_{\text {set }} F O_{2}$ (whence $M S_{2}$ ) but not $\exists_{\text {set }} F O_{1}$. However it is $F O_{1}^{T C}$ hence $M S_{1}$.

(3.1) Proposition: (1) Hamiltonicity is $\mathrm{MS}_{2}$ (and even $\exists_{\mathrm{set}} \mathrm{FO}_{2}^{\mathrm{TC}}$ ) but not $\mathrm{MS}_{1}$.

(2) The property that a directed graph has a directed spanning tree of outdegree at most 2 is $\mathrm{MS}_{2}$ (and even $\exists_{\mathrm{set}} \mathrm{FO}_{2}^{\mathrm{TC}}$ ) but not $\mathrm{MS}_{1}$.

Proof : (1) We know from Proposition (2.1) that Hamiltonicity is not $M S_{1}$. It is $M S_{2}$ by Propositions (2.1) and (2.3). We give here a simpler construction for the latter fact: $G$ is Hamiltonian iff it has at least 3 vertices and there exists a set of edges $X$ such that every vertex is incident to an edge of $X$, and there exist two adjacent vertices $x, y$ 


\section{BRUNO COURCELLE}

such that no edge belonging to $X$ links them, QuasiPath $(X, x, y)$ holds and every vertex in the graph is reachable from $x$ by an $X$-path. These conditions (for given $X$ ) are firstorder expressible, except the very last one which is $F O^{T C}$. This last condition is necessary because without it, we only express that $G$ is spanned by a union of disjoint cycles.

(2) Let $P(G)$ be the property of a simple directed graph $G$ that it has a directed spanning tree of outdegree at most 2 . It can be written :

There exists a subset $X$ of $\mathbf{E}_{G}$ such that every vertex is incident to an edge in $X$, every vertex is the origin of at most two edges in $X$ and $X$ is the set of edges of a directed tree $T \subseteq G$.

This property is in $\exists_{\mathrm{set}} F O{ }_{2}^{T C}$. Let us prove that it is not $M S_{1}$. For $n, m \geq 1$ we let $G_{n, m}$ be the graph with set of vertices $\{i \in \mathbb{Z} /-n \leq i \leq m\}$ and directed edges $(i, j)$ for $i<0$ and $j \in\{i+1,1,2, \ldots, m\}$. It is easy to see that $P\left(G_{n, m}\right)$ holds iff $m \leq n$. Hence $P$ is not $M S_{1}$. (The argument is similar to that of Proposition (2.1) using the fact that the language $\left\{a^{n} b^{m} / m \leq n\right\}$ is not regular).

(3.2) Question : Does there exist a $\exists_{\text {set }} \mathrm{FO}_{2}$ property that is not $\mathrm{MS}_{1}$ ?

Let $\mathbb{C}$ be a class of graphs. Let $\mathbb{L}$ be a fragment of $M S$, typically $\exists_{\text {set }} F O$, $\exists_{\text {set }} F O^{T C}$ or $M S$ itself. We say that edge quantifications are $\mathbb{L}$-eliminable for $\mathbb{C}$ if for every closed formula $\varphi$ in $\mathbb{L}_{2}$ one can construct a closed formula $\psi$ in $\mathbb{L}_{1}$ such that, for all graphs $G$ in $\mathbb{C}:|G|_{1} \vDash \psi$ iff $|G|_{2} \vDash \varphi$.

(3.3) Theorem ([6]): Let $\mathbb{L}$ be one of $\exists_{\mathrm{set}} \mathrm{FO}, \exists_{\mathrm{set}} \mathrm{FO}^{\mathrm{TC}}$ or MS. Edge quantifications are $\mathbb{L}$-eliminable for each of the following classes of directed (resp. undirected) graphs :

- the graphs of degree $\leq \mathrm{d}$ for any fixed $\mathrm{d}$,

- the planar graphs,

- the graphs of tree-width $\leq \mathrm{k}$ for any fixed $\mathrm{k}$.

In all cases the proof will consist in constructing $|G|_{2}$ from $|G|_{1}$ by means of logical formulas. (Since we only consider simple graphs, $|G|_{2}$ is uniquely defined up to isomorphism from $|G|_{1}$.)

Our objective will be to describe by monadic second-order formulas the transformation of the structure $|G|_{1}$ into the structure $|G|_{2}$. We need a definition concerning transformations of general relational structures. We will denote by $Q F$ the set of formulas without quantifiers.

We let $R$ and $Q$ be two sets of relation symbols. Let $\mathbb{L}$ be a fragment of $M S$. (We will use the notation $\mathbb{L}(R)$ to indicate the relevant set $R$ of relation symbols). An $(\mathbb{L}, R, Q)$-definition scheme is a tuple of formulas

$$
\triangle=\left\langle\delta,\left(\theta_{q}, b\right)_{q \in Q}, b \in\{0, \ldots, k\} \rho(q)>\right.
$$

of the following form : we fix $k, n \geq 0$ and two sets $\left\{Z_{O}, \ldots, Z_{k}\right\}$ and $\left\{Y_{1}, \ldots, Y_{n}\right\}$ of set variables. We let $\delta$ be a formula in $\mathbb{L}(R)$ with free variables in $\left\{Z_{o}, \ldots, Z_{k}\right.$, 
MONADIC SECOND-ORDER LOGIC

$\left.Y_{1}, \ldots, Y_{n}\right\}$. For every $q \in Q$ and $b \in\{0, \ldots, k\}^{m}, m=\rho(q)$ (the arity of the relation symbol $q$ ), we let $\theta_{q, b}$ be a formula in $\mathbb{L}(R)$ with free variables in $\left\{Z_{O}, \ldots, Z_{k}, Y_{1}, \ldots, Y_{n}\right.$, $\left.x_{1}, \ldots, x_{m}\right\}$.

For every $R$-structure $S=\left\langle\mathbf{D}_{S},\left(r_{S}\right)_{r \in R}\right\rangle$ for all subsets $Z_{o}, \ldots, Z_{k}, Y_{1}, \ldots, Y_{n}$ of $\mathbf{D}_{S}$ such that $\left(S, Z_{O}, \ldots, Z_{k}, Y_{1}, \ldots, Y_{n}\right) \quad \vDash \delta$ we define as follows a $Q$-structure $T=$ $\operatorname{def}_{\Delta}\left(S, Z_{o}, \ldots, Z_{k}, Y_{1}, \ldots, Y_{n}\right)$ :

$$
\begin{aligned}
& \mathbf{D}_{T}:=Z_{o} \times\{0\} \cup \ldots \cup Z_{k} \times\{k\} \\
& q_{T}\left(\left(d_{1}, i_{1}\right), \ldots,\left(d_{m}, i_{m}\right)\right): \Leftrightarrow i_{1}, \ldots, i_{m} \in\{0, \ldots, k\}, d_{1} \in Z_{i 1}, \ldots, d_{m} \in Z_{i m}, \text { and } \\
& \left(S, Z_{o}, \ldots, Z_{k}, Y_{1}, \ldots, Y_{n}, d_{1}, \ldots, d_{m}\right) \vDash \theta_{q, b} \quad \text { where } b=\left(i_{1}, \ldots, i_{m}\right) .
\end{aligned}
$$

We will say that the multivalued mapping associating with an $R$-structure $S$ the $Q$ structures of the form $\operatorname{def}_{\Delta}\left(Z_{O}, \ldots, Z_{k}, Y_{1}, \ldots, Y_{n}\right)$ such that $\left(S, Z_{o}, \ldots, Z_{k}, Y_{1}, \ldots, Y_{n}\right) \vDash \delta$ is an $\mathbb{L}$-definable transduction. The monadic first-order reductions used in [3] are FOdefinable transductions.

(3.4) Lemma : Let $\Delta$ be a (MS,R,Q) definition scheme. For every closed formula $\varphi$ $\in \mathrm{MS}(\mathrm{Q})$, one can construct a formula $\varphi^{\Delta} \in \mathrm{MS}(\mathrm{R})$ with free variables in $\left\{Z_{O}, \ldots, Z_{k}\right.$, $\left.Y_{1}, \ldots, Y_{n}\right\}$ such that, for every $\mathbb{R}$-structure $\mathrm{S}$ and for all sets $Z_{O}, \ldots, Z_{k}, Y_{1}, \ldots, Y_{n}$ $\subseteq \mathbf{D}_{\mathrm{S}}$

$$
\operatorname{def}_{\Delta}\left(S, Z_{O}, \ldots, Z_{k}, Y_{1}, \ldots, Y_{n}\right) \vDash \varphi \text { iff }\left(S, Z_{o}, \ldots, Z_{k}, Y_{1}, \ldots, Y_{n}\right) \vDash \varphi^{\Delta} .
$$

Furthermore, if $\Delta$ is an (FO,R,Q)-definition scheme and $\varphi \in \exists_{\mathrm{set}} \mathrm{FO}(\mathrm{Q})$ then $\varphi^{\Delta}$ $\in \exists_{\mathrm{set}} \mathrm{FO}(\mathrm{R})$. If, in addition, the formulas $\theta_{\mathrm{q}, \mathrm{b}}$ of $\Delta$ are without quantifiers and $\varphi \in$ $\exists_{\text {set }} \mathrm{FO}^{\mathrm{TC}}(\mathrm{Q})$ then $\varphi^{\Delta} \in \exists_{\text {set }} \mathrm{FO}^{\mathrm{TC}}(\mathrm{R})$.

Proof : Elementary manipulations of formulas. The idea is to replace a quantification of the form $\exists X . \varphi$ by quantifications of the form $\exists X_{0}, \ldots, X_{k+1}\left[X_{0} \subseteq Z_{0} \wedge \ldots \wedge X_{k+1} \subseteq\right.$ $\left.Z_{k}+1 \wedge \varphi^{\prime}\right]$ where $\varphi^{\prime}$ is an appropriate transformation of $\varphi$. We refer the reader to Courcelle [5] for details.

(In the last assertion we assume that the formulas $\theta_{\mathrm{q}, \mathrm{b}}$ are in $Q F$ (and not in $F O$ ) because in the transitive closure operators used in $F O^{T C}$, we assume that the formulas are existentially quantified.)

The various statements of Theorem (3.3) will be proved in the following way: for each class of graphs $\mathbb{C}$ we will construct a $(Q F,\{\mathbf{e d g}\},\{$ inc $\})$-definition scheme $\Delta=\langle\delta$, $\ldots>$ such that, for every $G \in \mathbb{C}$, there exist $Z_{O}, \ldots, Z_{k}, Y_{1}, \ldots, Y_{n}$ satisfying $\delta$, and for any such tuple: $\operatorname{def}_{\Delta}\left(|G|_{1}, Z_{O}, \ldots, Z_{k}, Y_{1}, \ldots, Y_{n}\right) \cong|G|_{2}$ (where $\cong$ denotes isomorphism of structures). Assuming that we have defined $\Delta$, the proof goes as follows. Given $\varphi \in \mathbb{L}_{2}$ we let $\varphi^{\Delta}$ be the corresponding formula in $\mathbb{L}_{1}$ (by Lemma (3.4)). Then a formula equivalent to $\varphi$ is $\exists Z_{O}, \ldots, Z_{k}, Y_{1}, \ldots, Y_{n}\left[\delta \wedge \varphi^{\Delta}\right]$ which is in $\mathbb{L}_{1}$ or can be transformed into a formula in $\mathbb{L}_{1}$ by some permutation of quantifiers.

Let $k$ be a positive integer and $G$ be a directed graph. A $k$-coloring of $G$ is a mapping $\gamma: \mathbf{V}_{G} \rightarrow\{1,2, \ldots, k\}$ such that $\gamma(v) \neq \gamma(w)$ whenever $(v, w) \in \mathbf{e d g}_{G}$. We say that $\gamma$ is semi-strong if $\gamma(v) \neq \gamma(w)$ whenever $v \neq w$ and there exists $x$ such that 


\section{BRUNO COURCELLE}

$(v, x)$ and $(w, x)$ belong to $\operatorname{edg}_{G}$. We denote by $\mathbf{S S}_{\mathbf{k}}$ the class of graphs having a semi-strong $k$-coloring.

(3.5) Proposition : For each $\mathrm{k} \geq 1$, there exists an FO-definable transduction associating $|\mathrm{G}|_{2}$ with $|\mathrm{G}|_{1}$ for every graph $\mathrm{G}$ in $\mathbf{S S}_{\mathbf{k}}$.

Proof : Assuming that we know a semi-strong coloring $\gamma$ (given by a $k$-tuple of sets of vertices $Y_{1}, \ldots, Y_{k}$ such that $\left.Y_{i}=\gamma^{-1}(i)\right)$, then we will represent an edge $e$ from $v$ to $w$ by the pair $\operatorname{rep}(e)=(w, \gamma(v))$. We let also $\operatorname{rep}(x)=(x, 0)$ for $x \in \mathbf{V}_{G}$. It follows that rep is a bijection of $\mathbf{V}_{G} \cup \mathbf{E}_{G}$ onto $Z_{o^{\times}}\{0\} \cup Z_{1} \times\{1\} \cup \ldots \cup Z_{k} \times\{k\}$ where $Z_{O}=$ $\mathbf{V}_{G}$ and $Z_{i}=\left\{w \in \mathbf{V}_{G} /\right.$ there is an edge $(v, w) \in \mathbf{E}_{G}$ for some $\left.v \in Y_{i}\right\}$ for $i \geq 1$. We let then $\theta_{\text {inc }, i, j, m}$ be the formula $x_{1}=x_{3} \wedge x_{2} \in Y_{i} \wedge x_{3} \in Z_{i} \wedge \operatorname{edg}\left(x_{2}, x_{3}\right)$ if $i$ $\neq 0, j=m=0$ and be the formula false if $i=0$ or $m \neq 0$. Whenever $Y_{1}, \ldots, Y_{k}, Z_{O}$ $, \ldots, Z_{k}$ are associated as above with a semi-strong $k$-coloring, $|G|_{2}$ is isomorphic by rep to the structure $\operatorname{def}_{\Delta}\left(|G|_{1}, Y_{1}, \ldots, Y_{k}, Z_{o}, \ldots, Z_{k}\right)$.

The formula $\delta$ intended to express that arbitrarily given sets $Y_{1}, \ldots, Y_{k}, Z_{o}, \ldots, Z_{k}$ are appropriate can be defined as the conjunction of the following first-order conditions :

1) $Y_{1}, \ldots, Y_{k}$ form a partition of $\mathbf{V}_{G}$,

2) any two adjacent vertices are not in the same set $Y_{i}$ for any $i$,

3) any two distinct vertices $x, y$ such that $\operatorname{edg}_{G}(x, u)$ and $\operatorname{edg}_{G}(y, u)$ for some $u$ are not in the same set $Y_{i}$ for any $i$,

4) $Z_{o}=\mathbf{V}_{G}$,

5) for every $i=1, \ldots, k$ and $x \in \mathbf{V}_{G}: x \in Z_{i}$ iff there is $y \in Y_{i}$ such that $\operatorname{edg}_{G}(y, x)$ holds.

It follows that $\Delta$ is a $(F O,\{\mathbf{e d g}\},\{$ inc $\})$-definition scheme and that a graph $G$ is in $\mathbf{S S}_{\mathbf{k}}$ iff there exist a $2 k$-tuple of sets $\left(Y_{1}, \ldots, Y_{k}, Z_{O}, \ldots, Z_{k}\right)$ such that $\left(|G|_{1}, Y_{1}\right.$, $\left.\ldots, Y_{k}, Z_{o}, \ldots, Z_{k}\right) \vDash \delta$. Furthermore, for any such tuple, $\operatorname{def}_{\Delta}\left(|G|_{1}, Y_{1}, \ldots, Y_{k}\right.$, $\left.Z_{o}, \ldots, Z_{k}\right)$ is isomorphic to $|G|_{2}$ by rep.

One next aim is to extend this result to certain undirected graphs by equipping them with an adequate orientation that we will define by $Q F_{1}$ formulas (and not by $F O{ }_{2}^{T C}$ formulas as in Section 2).

Let $C$ be a $k$-tournament, i.e., a directed graph with set of vertices $\{1, \ldots, k\}$ and for every $1 \leq i<j \leq k$ an edge either from $i$ to $j$ or from $j$ to $i$. Let $G$ be an undirected graph, and $\gamma: \mathbf{V}_{G} \rightarrow\{1, \ldots, k\}$ be a $k$-coloring. We denote by $G(C, \gamma)$ the orientation of $G$ obtained by directing an edge $\{u, v\}$ from $u$ to $v$ iff $(\gamma(u), \gamma(v))$ is an edge of $C$. We let $\mathbb{G}(C)$ denote the class of graphs $H$ of the form $G(C, \gamma)$ for some $G$ and $\gamma$, such that $\gamma$ is a semi-strong coloring of $H$. We let $\mathbb{G}(k)=U_{\{\mathbb{G}}(C) / C$ is a $k$-tournament $\}$.

(3.6) Proposition : For each $\mathrm{k}$, the result of Proposition (3.5) holds for the classes $\operatorname{und}(\mathbb{G}(\mathrm{k}))$ and und $^{-1}(\operatorname{und}(\mathbb{G}(\mathrm{k})))$.

Proof: The result holds for each class und $(\mathbb{G}(C))$ since an appropriate orientation is definable from a coloring $\gamma$ of a graph $G$ in und $(\mathbb{G}(C))$ where $\gamma$ is given as in (3.5) by sets $Y_{1}, \ldots, Y_{k}$. If the result holds for two classes, it holds for their union. The result follows thus for und $(\mathbb{G}(k))$. 
MONADIC SECOND-ORDER LOGIC

We now consider the case of a graph $H$ in und $^{-1}(\operatorname{und}(\mathbb{G}(k)))$. By reversing the orientation of a set $X$ of edges of $H$ we obtain a graph $G$ in $\mathbb{G}(k)$. Let $Y_{1}, \ldots, Y_{k}$, $Z_{O}, \ldots, Z_{k}$ be a tuple associated with $G$ as in (3.5). Since a set of edges $X$ of und(G) can be specified by $k$ sets of vertices $X_{1}, \ldots, X_{k}$ of und $(G)$ (equivalently, of $H$ ), we can define, by $Q F$-definition schemes, $|G|_{1}$ from $|H|_{1},|G|_{2}$ from $|G|_{1}$ and $|H|_{2}$ from $|G|_{2}$ in terms of the sets $Y_{1}, \ldots, Y_{k}, Z_{o}, \ldots, Z_{k}, X_{1}, \ldots, X_{k}$. One can combine these definitions and one gets finally a definition scheme defining $|H|_{2}$ from $\left(|H|_{1}, Y_{1}, \ldots\right.$, $\left.Y_{k}, Z_{o}, \ldots, Z_{k}, X_{1}, \ldots, X_{k}\right)$ where $Y_{1}, \ldots, Y_{k}, Z_{o}, \ldots, Z_{k}, \ldots, X_{1}, \ldots, X_{k}$ are appropriately chosen. That they actually are, can be verified by a first-order formula. We refer the reader to [6] for more details.

Proof of Theorem (3.3): It is proved in [6, Lemmas 5.1 and 5.2] that every undirected graph of degree $\leq d$ is in und $(\mathbb{G}(k))$ where $k=(m+1)^{2} 3^{m}$ and $m=d^{2}$. Proposition 6.7 of the same paper shows that every undirected graph of tree-width $\leq k$ belongs to und $\left(\mathbb{G}\left(3^{k}\right)\right)$. Improving a result of [6], Raspaud and Sopena proved ([22, Thm 2]) that every undirected planar graph is in und $(\mathbb{G}(320))$. The result follows then immediately from Proposition (3.6). These lemmas from [6, 22] are purely graph theoretical. Their proofs make no use of logic.

\section{It is easier to forbid than to build}

Certain classes of graphs can be defined in two different ways: either "positively" by expressing that a graph has a certain structure or "negatively" by expressing that a graph has no subgraph of a certain kind. We consider two such classes, the class of chordal graphs and the class of 3-connected planar graphs: for both cases we obtain that the "negative" definition is simpler than the "positive" one : the former is in $\exists_{\text {set }} F O_{1}$ whereas the latter is in $\exists_{\mathrm{set}} F O_{2}^{T C}$.

\section{Chordal graphs}

An undirected graph is chordal iff it has no induced subgraph which is a cycle with at least 4 vertices. Chordal graphs have particular algorithmic properties (see Gavril [17]). However, these properties are not based on this definition which is "negative" and says little on the structure of chordal graphs, but on an equivalent one showing that chordal graphs have a certain characteristic "tree-structure".

By the definition, Non-Chordality is characterized by the following $\exists_{\text {set }} F O_{1}$ property (see Section 1 for QuasiPath'):

$$
\begin{aligned}
& \exists X[\exists x, y, z(x \neq y \wedge x \in X \wedge y \in X \wedge \neg z \in X \wedge \neg \operatorname{edg}(x, y) \wedge \operatorname{edg}(x, z) \wedge
\end{aligned}
$$

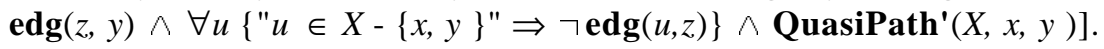

We now consider an alternative characterization exhibiting the tree-structure of chordal graphs which is important for algorithmic purposes. A directed chordal graph is a directed acyclic graph $G$ such that, for every two edges $(x, y)$ and $\left(x^{\prime}, y\right)$ in $G, x$ and $x^{\prime}$ are adjacent. It is known from Rose [23] that a graph $G$ is chordal iff it is $\operatorname{und}(H)$ for some directed chordal graph $H$. DirectedChordality is clearly $F O{ }_{1}^{T C}$. We will prove 


\section{BRUNO COURCELLE}

that Chordality is $\exists_{\mathrm{set}} F O_{2}^{T C}$. The corresponding formula will be "constructive": it will specify, (when it holds) an orientation of the considered graph $G$ making it into a directed chordal graph.

(4.1) Lemma : A connected directed acyclic graph $\mathrm{G}$ is directed chordal iff it has a directed spanning tree $\mathrm{T}$ such that for every edge $(\mathrm{x}, \mathrm{y})$ not in $\mathrm{T}$ we have $\mathrm{x}<\mathrm{T}, \mathrm{y}$, and for every $\mathrm{z}$ with $\mathrm{x}<\mathrm{T} \mathrm{z}<\mathrm{T}$ y we have an edge in $\mathrm{G}$ from $\mathrm{z}$ to $\mathrm{y}$ and one from $\mathrm{x}$ to $\mathrm{z}$.

Before doing the proof, we recall some definitions. A tree-decomposition of a graph $G$ is a pair $(T, f)$ consisting of a tree $T$ and a mapping $f=\mathbf{V}_{T} \rightarrow P\left(\mathbf{V}_{G}\right)$ satisfying the following conditions :

i) every vertex belongs to $f(x)$ for some $x$,

ii) every edge has its two ends in $f(x)$ for some $x$,

iii) for every $y \in \mathbf{V}_{G},\left\{x \in \mathbf{V}_{T} / y \in f(x)\right\}$ is a connected subgraph of $T$.

The width of $(T, f)$ is the maximal cardinality of the sets $f(x)$ minus 1 . The treewidth of a graph is the minimal width of its tree-decompositions.

Proof of Lemma 4.1: "If". Let $G$ be a directed acyclic graph with a spanning tree $T$ satisfying the condition of the statement. Let $(x, y)$ and $\left(x^{\prime}, y\right)$ be two edges. Then we have $x<_{T} y$ and $x^{\prime}<_{T} y$ and we must have $x<_{T} x^{\prime}$ or $x^{\prime}<_{T} x$. Assume the first: we have thus an edge from $x$ to $x^{\prime}$ since $x<_{T} x^{\prime}<_{T} y$.

"Only if". Let $G$ be directed chordal and connected. Consider a vertex $v$ of outdegree 0 in $G$. Let $\left\{x_{1}, \ldots, x_{k}\right\}$ be the vertices adjacent to $v$. They form a clique $C$. It is easy to see that every clique in $G$ is linearly ordered by the transitive and reflexive closure of $\operatorname{edg}_{G}$. Assume $x_{k}$ is the maximal element of $C$ with respect to this partial order. By induction we have that $G-v$ is directed chordal, we can assume that $T^{\prime}$ is a directed spanning tree of $G-v$ as desired and we build $T$ by adding to $T^{\prime}$ the directed edge $\left(x_{k}, v\right)$.

The remaining verifications are easy.

We can thus express that a connected undirected graph $G$ is chordal as follows :

$\exists Y$ [" $Y$ is a set of edges" $\wedge \exists u("(Y, u)$ is a dfs spanning tree" $\wedge$

"for every edge $\{x, y\}$ not in $Y$ we have $x<_{(Y, u)} y$ or $y<_{(Y, u)} x$ " $\wedge$

"for every vertex $z$ such that $x<_{(Y, u)} z<_{(Y, u)} y,\{x, z\}$ and $\{z, y\}$ are edges")].

This definition is clearly in $\exists_{\mathrm{set}} F O{ }_{2}^{T C}$. The extension to graphs that are not connected is immediate by using dfs forests, as in the proof of Proposition (2.2).

Finally, we may observe that we obtain in this way a tree-decomposition of $G$. By taking the tree $T=(Y, u)$ of (3) and the mapping $f$ such that $f(x)=\{x\} \cup\{y /(y, x)$ is an edge of $T$ \} one obtains a tree-decomposition of $G$. All "boxes" $f(x)$ of this treedecomposition are cliques of $G$.

It is easy to check that a graph is chordal iff it has a tree-decomposition all boxes of which are cliques.

A tree-decomposition $(T, f)$ of a graph $G$ will be represented by the structure $|(T, f)|_{2}$ $=\left\langle\mathbf{V}_{G} \cup \mathbf{E}_{G} \cup \mathbf{V}_{T}\right.$, inc $\left._{G}, \mathbf{e d g}_{T}, \boldsymbol{b o x}_{T}\right\rangle$ where $\operatorname{box}_{T}(x, y)$ holds iff $x \in \mathbf{V}_{T}, y \in$ $f(x)$ (we assume that $\left.\mathbf{V}_{T} \cap\left(\mathbf{V}_{G} \cup \mathbf{E}_{G}\right)\right)=\varnothing$. 
MONADIC SECOND-ORDER LOGIC

(4.2) Corollary: One can build a definition scheme $\Delta=\left\langle\delta, \ldots>\right.$ with parameters $\mathrm{Z}_{0}$, $\mathrm{Z}_{1}, \mathrm{Y}_{1}$, and formula $\delta$ in $\mathrm{FO}_{2}^{\mathrm{TC}}$ such that, for every undirected connected graph $\mathrm{G}$,

$|\mathrm{G}| 2 \vDash \exists \mathrm{Z}_{0}, \mathrm{Z}_{1}, \mathrm{Y}_{1}[\delta]$ iff $\mathrm{G}$ is chordal

and for every triple $\mathrm{Z}_{0}, \mathrm{Z}_{1}, \mathrm{Y}_{1}$ satisfying $\delta$ the structure $\operatorname{def}_{\Delta}\left(|\mathrm{G}| 2, \mathrm{Z}_{0}, \mathrm{Z}_{1}, \mathrm{Y}_{1}\right)$ is isomorphic to a structure representing a tree-decomposition of $\mathrm{G}$.

Proof : We let $\delta$ express that $Z_{0}=\mathbf{V}_{G} \cup \mathbf{E}_{G}, Z_{1}=\mathbf{V}_{G}, Y_{1}$ is a set of edges of $G$, $Y_{2}=\{u\}, u \in \mathbf{V}_{G}$, and $\left(Y_{1}, u\right)$ witnesses that $G$ is chordal (see (3) after Lemma (4.1)). The remaining details are easy to fill in.

\section{Planar graphs}

We have seen that Non-Planarity is a $\exists_{\text {set }} F O_{1}$ property. Our goal here is to express Planarity by a formula in $\exists_{\mathrm{set}} F \mathrm{O}_{2}^{T C}$ which specifies, when it holds, a planar embedding of the considered graphs. We will consider graphs in $\mathbb{G}$, the class of connected undirected graphs.

\section{Part 1: Logical representation of planar drawings.}

Let $G$ be a planar graph in $\mathbb{G}$. A planar drawing of $G$ can be defined from a triple ( $r$, $T, S)$ where $(T, r)$ is a spanning tree of $G$ and $S \subseteq \mathbf{E}_{G^{\times}} \mathbf{E}_{G}$ is a relation which defines a strict linear order on each set $\mathbf{E}_{G}(x), x \in \mathbf{V}_{G}$ (we denote by $\mathbf{E}_{G}(x)$ the set of edges incident with $x$ ).

The drawing is done from $(r, T, S)$ as follows: one first draws $(T, r)$ by placing the edges going out of a vertex $x$ from left to right according to the order on $\mathbf{E}_{G}(x)$ defined by $S$; one places then the edges not in $T$ in such a way that the relative order of incidence around a vertex $x$ is determined by the restriction of $S$ to $\mathbf{E}_{G}(x)$. This is possible without crossings if $S$ satisfies condition $(C)$ stated below, with help of some notation.

We let $B=\left\{(e, x) / e \in \mathbf{E}_{G^{-T}}, x \in \mathbf{V}_{G}, x\right.$ is incident with $\left.e\right\}$. We let $<_{S}$ be the strict linear order on $B$ defined as follows :

$(e, x)<_{S}\left(e^{\prime}, x^{\prime}\right)$ iff $\left(e_{1}, e_{2}, \ldots, e_{k}, e\right)<_{S}^{\operatorname{lex}}\left(e^{\prime}{ }_{1}, \ldots, e_{m}^{\prime}, e^{\prime}\right)$

where $\left(e_{1}, e_{2}, \ldots, e_{k}\right)$ is the sequence of edges of the unique path in $T$ from $r$ to $x$ and similarly for $\left(e^{\prime} 1, \ldots, e_{m}^{\prime}\right)$ and $x^{\prime}$, and $<_{S}^{\text {lex }}$ is the lexicographic ordering associated with $S$.

Condition $C$ is now the following:

$(C)$ : there are no two edges $e=\{x, y\}$ and $e^{\prime}=\left\{x^{\prime}, y^{\prime}\right\}$ in $\mathbf{E}_{G}-\mathbf{E}_{T}$ such that:

$$
(e, x)<_{S}\left(e^{\prime}, x^{\prime}\right)<_{S}(e, y)<_{S}\left(e^{\prime}, y^{\prime}\right) .
$$


That this condition ensures the possibility of placing the edges of $\mathbf{E}_{G}-T$ without crossings can be explained with the help of an example.

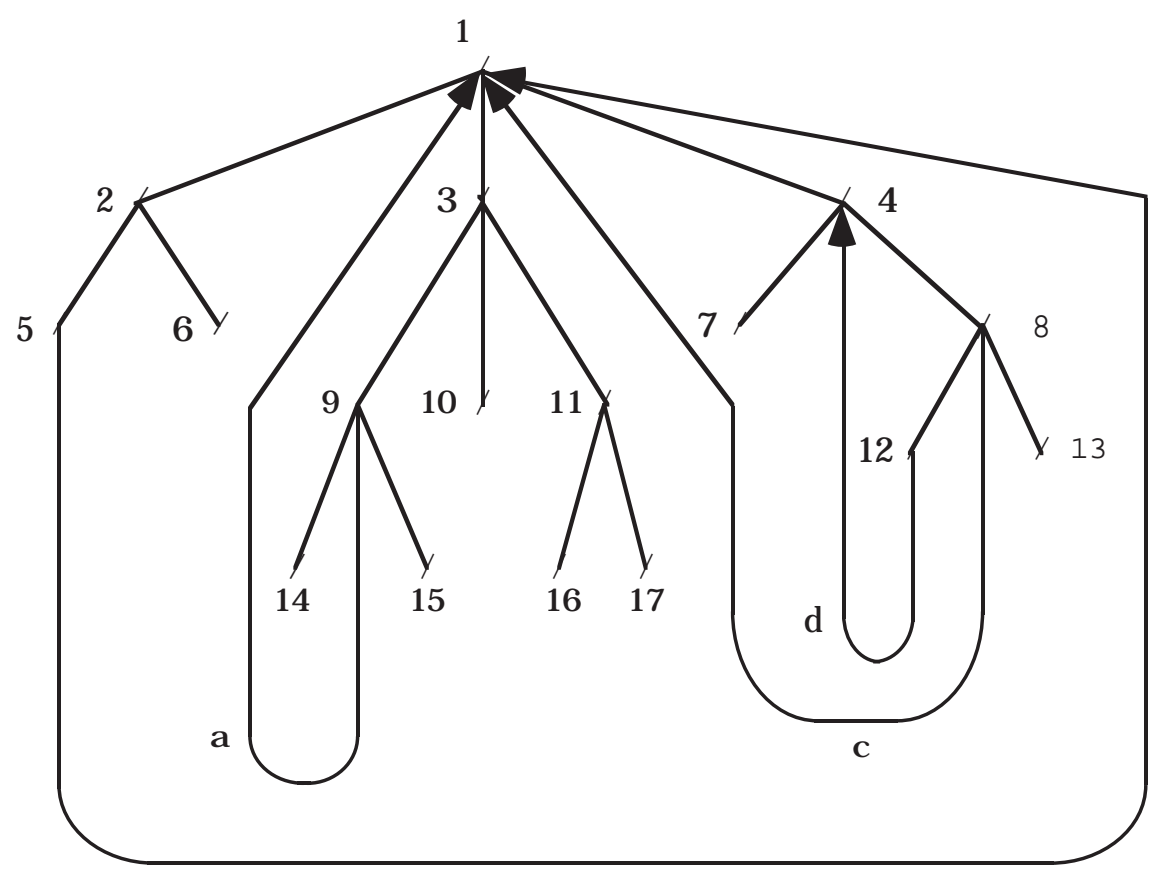

b

Figure 1

Example: Consider the graph $G$ of Figure 1 with vertices numbered from 1 to 17 and $\mathbf{E}_{G}-\mathbf{E}_{T}=\{a, b, c, d\}$. We assume that $S$ induces the following linear orders :

$$
\begin{gathered}
\{1,2\}<a<\{1,3\}<c<\{1,4\}<b \text { on } \mathbf{E}_{G}(1), \\
\{1,3\}<\{3,9\}<\{3,10\}<\{3,11\} \text { on } \mathbf{E}_{G}(3), \\
\{1,4\}<\{4,7\}<d<\{4,8\} \text { on } \mathbf{E}_{G}(4), \\
\{1,8\}<\{8,12\}<c<\{8,13\} \text { on } \mathbf{E}_{G}(8), \\
\{3,9\}<\{9,14\}<a<\{9,15\} \text { on } \mathbf{E}_{G}(9) .
\end{gathered}
$$

The order $<_{S}$ on $B$ is then:

$$
(b, 5)<<_{S}(a, 1)<<_{S}(a, 9)<<_{S}(c, 1)<<_{S}(d, 4)<<_{S}(d, 12)<<_{S}(c, 8)<<_{S}(b, 1) .
$$

A drawing can be done as follows: one cuts in two parts each edge of $\mathbf{E}_{G^{-}} \mathbf{E}_{T}$; one draws $T$ and these half-edges according to $S$; Condition $(C)$ guarantees that the two halves of any edge in $\mathbf{E}_{G}-\mathbf{E}_{T}$ can be linked without creating any crossing.

We define a map of a graph $G$ as a triple $D=(r, T, S)$ satisfying condition (C). Such a map will be defined by the structure: 
MONADIC SECOND-ORDER LOGIC

$|D|_{2}:=\left\langle\mathbf{V}_{G} \cup \mathbf{E}_{G}\right.$, inc $_{G}, \operatorname{root}_{G}$, tree-edge $\left._{G}, \mathbf{s}_{G}\right\rangle$

where $\operatorname{root}_{G}(x)$ holds iff $x=r$, tree-edge $G^{(e)}$ holds iff $e \in T$ and $\mathbf{s}_{G}(x, y)$ holds iff $(x, y) \in S$.

Part 2: Defining a map from a dfs tree and two sets of edges.

We now establish that for planar 3-connected graphs $G$ in $\mathbb{G}$ the required objects $T$ and $S$ can be specified by formulas in $\exists_{\mathrm{set}} F \mathrm{O}_{2}^{T C}$. (A graph is 3-connected if the property 2-Separability $(x, y)$ holds for no two vertices $x, y$.)

We first define a construction of $T$ from a planar drawing of $G$. We take an drawing in the closed half-plane with a single vertex, say $r$, on the border. Let $(T, r)$ be a dfs spanning tree of $G$. We will denote by $H$ the directed graph $G\left(T, r, \mathbf{E}_{G^{-}} T\right.$ ) (see Section 2). For every edge $(x, y) \in \mathbf{E}_{G}-T$ we have $y<_{T} x$. We call it a left-edge if it reaches $y$ from the left of the branch in $T$ going from $r$ to $x$. We call it a right-edge otherwise. We let $L$ be the set of left-edges and $R$ be the set of right-edges. (On Figure 1, $a$ from 9 to $1, d$ from 12 to 4 and $c$ from 8 to 1 are left-edges, $b$ from 5 to 1 is a right-edge; the graph $G$ is not 3-connected).

We will prove that if $G$ is 3 -connected its unique embedding in the plane can be defined from any 4-tuple $(r, T, L, R) \in \mathbf{V}_{G} \times P\left(\mathbf{E}_{G}\right)^{3}$ satisfying some conditions C1-C3 listed below, which hold if $r, T, L, R$ are associated as explained above with a plane drawing of $G$, and that are expressible by formulas in $F O{ }_{2}^{T C}$. Here are conditions $\mathrm{C} 1$ C2:

$\mathrm{C} 1:(T, r)$ is a dfs spanning tree of $G$,

$\mathrm{C} 2:(T, L, R)$ is a partition of $\mathbf{E}_{G}$.

Assuming them, we let $H:=G\left(T, r, \mathbf{E}_{G}-T\right)$; we will consider $T, L, R$ as subsets of $\mathbf{E}_{H}$ hence as sets of directed edges. We will say that a path from $w$ to $x$ is a left path (resp. a right path) if its last edge is in $L$ (resp. in $R$ ). We will write $\mathbf{U}_{L}(e, x)$ if $e$ is the first edge of a left path from some vertex $w$ (the origin of $e$ ) to $x$. We define similarly $\mathbf{U}_{R}(e, x)$ with right paths. Finally, for $e=(w, y), e=\left(w, y^{\prime}\right) \in \mathbf{E}_{H}$, we let $Q\left(e, e^{\prime}\right)$ hold iff for some $x, x^{\prime}<_{T} w$ we have :

either $\mathbf{U}_{L}(e, x)$ and $\mathbf{U}_{L}\left(e^{\prime}, x^{\prime}\right)$ and $x^{\prime}<_{T} x$,

or $\mathbf{U}_{R}(e, x)$ and $\mathbf{U}_{R}\left(e^{\prime}, x^{\prime}\right)$ and $x^{\prime}>_{T} x$,

or $\mathbf{U}_{L}(e, x)$ and $\mathbf{U}_{R}\left(e^{\prime}, x^{\prime}\right)$.

Our third condition now reads :

C3 : for every two edges $e, e^{\prime}$ if $Q\left(e, e^{\prime}\right)$ holds then $Q\left(e^{\prime}, e\right)$ does not.

Conditions C1-C3 hold if $r, T, L, R$ are associated as explained above with a plane drawing of $G$. For proving the converse, namely that a map $(r, T, S)$ can be defined from them if they hold, we need some lemmas :

(4.3) Lemma : Let $\mathrm{H}$ be a 3-connected directed graph. Let $\mathrm{T}, \mathrm{L}, \mathrm{R}$ satisfy conditions C1-C3. For every vertex $\mathrm{w}$, the relation $\mathrm{Q}$ defines a strict linear order on the set of edges with origin $\mathrm{w}$.

We will extend $Q$ into a binary relation $S$ which is a strict linear order on each set $\mathbf{E}_{G}(w)$. We will denote by $\operatorname{org}(e)$ the origin of an edge e and by $\operatorname{tgt}(e)$ its target. If $e \in$ 
BRUNO COURCELLE

$L \cup R$ and $w$ is a vertex with $w \geq_{T} \operatorname{tgt}(e)$ we let $O R G(e, w)$ be the unique edge in $T$ with origin $w$ which is on a directed path from $w$ to $\operatorname{tgt}(e)$ the last edge if which is $e$. (If there is no such path then $\operatorname{ORG}(e, w)$ is undefined). We now define $S$ :

$S\left(e, e^{\prime}\right): \Leftrightarrow e \neq e^{\prime}, e, e^{\prime} \in \mathbf{E}_{G}(w)$ for some (necessarly unique) $w$, and either $e \in T$ and $\operatorname{tgt}(e)=w$

or $w=\operatorname{org}(e)=\operatorname{org}\left(e^{\prime}\right)$ and $Q\left(e, e^{\prime}\right)$

or $e^{\prime}=O R G(e, w)$ and $e^{\prime} \in R$

or $e=O R G\left(e^{\prime}, w\right)$ and $e \in L$

or $w=\operatorname{org}(e)$ and $Q\left(e, O R G\left(e^{\prime}, w\right)\right)$

or $w=\operatorname{org}\left(e^{\prime}\right)$ and $Q\left(\right.$ ORG $\left.(e, w), e^{\prime}\right)$

or $w=\operatorname{tgt}(e)=\operatorname{tgt}\left(e^{\prime}\right), e, e^{\prime} \in L \cup R$ and we have:

either $Q\left(\right.$ ORG $\left.(e, w), O R G\left(e^{\prime}, w\right)\right)$

or $O R G(e, w)=O R G\left(e^{\prime}, w\right)$ and for some $w^{\prime}>_{T} w$ we have

$f=O R G\left(e, w^{\prime}\right) \neq O R G\left(e^{\prime}, w^{\prime}\right)=f^{\prime}$ and

either $Q\left(f, f^{\prime}\right), e \in L, e^{\prime} \in R$

or $Q\left(f^{\prime}, f\right), e, e^{\prime} \in R$

or $Q\left(f^{\prime}, f\right), e, e^{\prime} \in L$.

(4.4) Lemma: Under the hypothesis of Lemma (4.3) the relation $\mathrm{S}$ defines a linear ordering of each set $\mathbf{E}_{G}(\mathrm{x})$ for $\mathrm{x} \in \mathbf{V}_{G}$ and the pair $(\mathrm{T}, \mathrm{S})$ satisfies condition $\mathrm{C}$.

This lemma and the previous one will be proved in a forthcoming paper.

(4.5) Theorem: The planarity of a 3-connected graph can be expressed by a formula $\psi$ of the form $\exists \mathrm{X}, \mathrm{T}, \mathrm{L}, \mathrm{R} . \varphi$ where $\varphi$ is $\mathrm{FO}_{2}^{\mathrm{TC}}$. When $\psi$ holds, one can define from $\mathrm{X}, \mathrm{T}$, $\mathrm{L}, \mathrm{R}$ satisfying $\varphi$ and by a $\mathrm{FO}_{2}^{\mathrm{TC}}$ formula, a binary relation $\mathrm{S}$ on $\mathbf{E}_{G}$ which satisfies the condition $\mathrm{C}$ and from which a planar drawing of $\mathrm{G}$ can be defined.

(4.6) Corollary: One can build a definition scheme $\Delta$ that defines for every 3connected planar graph $\mathrm{G}$ given by $|\mathrm{G}|_{2}$ a structure $|\mathrm{D}|_{2}$ representing a map $\mathrm{D}$ of $\mathrm{G}$.

Corollary (4.6) does not extend to general, even 2-connected, planar graphs. Consider for instance the 2-connected graphs shown in Figure 2, for arbitrary large sets $X$.

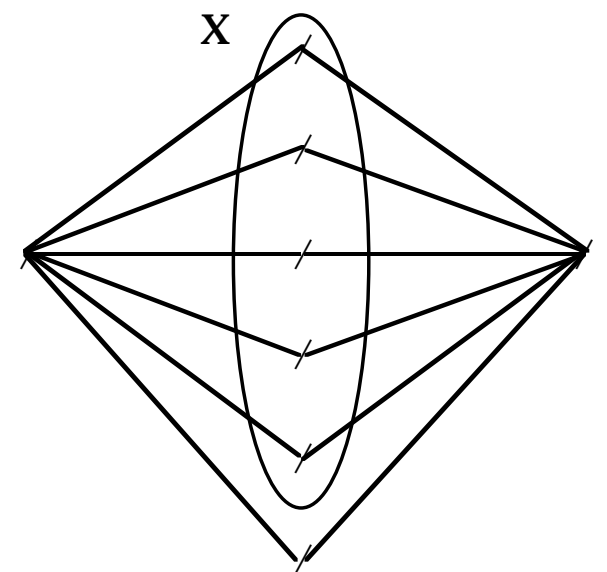

Figure 2 
MONADIC SECOND-ORDER LOGIC

Defining a planar drawing of this graph is equivalent to defining a linear order of the set of vertices $X$. If one could define such a drawing by $M S$ formulas one could define by $M S$ formulas a linear order on any set, which is not possible (we skip details ; see [7]).

\section{Overview of further topics and open problems}

We first discuss two extensions of $M S$. The first extension is called counting monadic second-order logic (CMS), and is obtained from $M S$ by the use of atomic formulas of the form $\operatorname{card}_{p}(X), p \geq 2$, meaning that the set $X$ has cardinatlity $\lambda p$ for some $\lambda \in \mathbb{N}$. CMS is an extension of $M S$ which is strictly more expressive. However, in a structure where a linear order is definable by an MS formula, every formula of $C M S$ can be transformed into an equivalent one in $M S$ (see [4]).

Another extension of $M S$ is $M S(\leq)$, i.e., $M S$ logic in presence of a built-in linear order. (A property $P$ of a structure $S$ is $M S(\leq)$ iff it is equivalent to an $M S$ property $Q$ of $(S, \leq)$, i.e., of the structure $S$ augmented with a binary relation $\leq$ which is a linear order of $\mathbf{D}_{S}$, and such that for any two linear orders $\leq$ and $\leq$ on $\mathbf{D}_{S}$ it holds that $Q(S, \leq)$ iff $Q\left(S, \leq^{\prime}\right)$.) Every $C M S$ property is $M S(\leq)$.

(5.1) Conjecture ([9]) : There exists a $\mathrm{MS}(\leq)$ property which is not CMS.

Seese [24] has proved that if a set of graphs is such that its $M S_{2}$-theory is decidable then its elements have uniformly bounded tree-width. (See the beginning of Section 4 for the definition of tree-width.)

(5.2) Conjecture ([24]): If a set of graphs $\mathrm{L}$ has a decidable $\mathrm{MS}_{1}$-theory, then $\mathrm{L}$ $\subseteq \operatorname{def}_{\Delta}(\mathrm{K})$ for some MS-definition scheme $\Delta$ where $\mathrm{K}$ is the set of binary trees.

An equivalent form of this conjecture is discussed in [7], and proved for certain sets of chordal graphs and for any class of directed graphs $L$ closed under arbitrary changes of edge directions (i.e., such that $L=$ und $\left.^{-1}(\mathbf{u n d}(L))\right)$.

We showed in Section 4 (Corollary 4.2) how a tree-decomposition of a chordal graph can be defined by $M S$-formulas.

(5.3) Conjecture ([5]): For every k one can construct an MS-definable transduction associating with every graph $\mathrm{G}$ of tree-width at most $\mathrm{k}$ a tree-decomposition of it of width at most $\mathrm{k}$.

The case $k=1$ is fairly obvious, the cases $k=2$ and $k=3$ are proved respectively in [5] and [19]. The $M S$ definition of tree-decompositions of width $g(k)$ for graphs of treewidth at most $k$ (where $g$ is a fixed function such that $g(k) \geq k$ for all $k$ ) would yield a weak form of this conjecture and prove the following one :

(5.4) Conjecture ([5]): For each k, every recognizable set of graphs of tree-width $\leq \mathrm{k}$ is CMS-definable. 


\section{BRUNO COURCELLE}

This would generalize the similar result for words and trees (see [27, Thm 3.2], [4]). We only recall here that recognizability for sets of graphs is defined algebraically in terms of finite congruences ([4]) and not in terms of automata.

Every graph $G$ has a unique modular decomposition [9], i.e., a unique hierarchical structuring based on the substitution of a graph for a vertex in a graph. This structuring is $M S$-definable in the structure $\left(|G|_{1}, \leq\right)$ where $\leq$ is an arbitrary (built-in) linear ordering of $\mathbf{V}_{G}$.

(5.5) Conjecture [9] : The modular decomposition of a graph G is not MS-definable in $|G|_{1}$.

Some of our results hold for infinite graphs: Propositions (2.2) and (2.3), because infinite graphs have dfs spanning forests and Theorems (3.3) and (3.6) because the coloring lemmas of Section 3 extend from finite graphs to infinite ones.

Acknowledgements: I dedicate this work to the memory of P. Kannellakis who died in a plane accident in 1995. I thank C. Lautemann and H. Straubing for their comments on the first version of this article.

\section{References}

1. M. Ajtai, R. Fagin, Reachability is harder for directed than for undirected finite graphs, J. of Symbolic Logic 55 (1990) 113-150.

2. S. Arnborg, J. Lagergren, D. Seese, Easy problems for tree decomposable graphs, J. of Algorithms 12 (1991) 308-340.

3. S. Cosmadakis, Logical reducibility and monadic NP, Proceedings of 33rd IEEE Symp. on Foundations of Computer Science, 1993, pp. 52-61.

4. B. Courcelle, The monadic second-order logic of graphs I: Recognizable sets of finite graphs, Information and Computation 85 (1990) 12-75.

5. B. Courcelle, The monadic second-order logic of graphs V: On closing the gap beween definability and recorgnizability, Theoret. Comput. Sci. 80 (1991) 153-202.

6. B. Courcelle, The monadic second-order logic of graphs VI: On several representations of graphs by relational structures, Discrete Applied Mathematics 54 (1994) 117-149.

7. B. Courcelle, The monadic second-order logic of graphs VIII: Orientations, Annals Pure Applied Logic, 72 (1995) 103-143.

8. B. Courcelle, Monadic second-order definable graph transductions: a survey, Theoret. Comput. Sci. 126(1994) 53-75.

9. B. Courcelle, The monadic second order logic of graphs $X$ : Linear orders, Theoret. Comput. Sci. 160 (1996) 87-143.

10 B. Courcelle, M. Mosbah, Monadic second-order evaluations on tree-decomposable graphs, Theoret. Comput. Sci. 109 (1993) 49-82.

11. E. Emerson, Temporal and modal logic, in "Handbook of Theoretical Computer Science, vol. B", J. Van Leeuwen ed., Elsevier, 1990, pp. 995-1072.

12.R. Fagin, Generalized first-order spectra and polynomial time recognizable sets, in "Complexity of Computation" R. Karp ed., SIAM-AMS proceedings 7 (1974) 43-73.

13. R. Fagin, Monadic generalized spectra, Z. für Math-Logic Grundlagen Math. 21 (1975) 89-96.

14. R. Fagin, Finite model theory - a personal perspective, Theoret. Comput. Sci. 116 (1993) 3-31.

15. R. Fagin, L. Stockmeyer, M. Vardi, On monadic NP vs. monadic co-NP, Information and Computation 120 (1995) 78-92.

16. H. Gaifman, On local and non local properties, Logic Colloquium'81, J. Stern ed., NorthHolland, 1982, pp. 105-135. 


\section{MONADIC SECOND-ORDER LOGIC}

17. F. Gavril, Algorithms for minimum coloring, maximum clique, minimum covering by cliques and maximum independent set of a chordal graph, SIAM J. Comput. 1 (1972)180187.

18. N. Immerman, Languages that capture complexity classes, SIAM J. Comput. 16 (1987) $760-778$.

19. D. Kaller, Definability equals recognizability of partial 3-trees, Proceedings of WG'96, Como, Italy, Lec Notes in Computer Science, 1996, to appear.

20. J. Makowsky, Model theory and computer science: an appetizer, Chapter I.6 of "Handbook of Logic in Computer Science, Vol. 1", S. Abramsky et al. eds., Oxford University Press, 1992.

21. J. Makowsky, Y. Pnueli, Arity vs. Alternation in second-order logic, in "Logical Foundations of Computer Science", A. Nerode, Y. Matiyasevich eds., LNCS, vol. 813 (1994) 240-252.

22. A. Raspaud, E. Sopena, Good and semi-strong colorings of oriented planar graphs, Inf. Proc. Letters 51 (1994) 171-174.

23. D. Rose, Triangulated graphs and the elimination process, J. Math. Anal. Appl. 32 (1970) 597-609.

24. D. Seese, The structure of the models of decidable monadic theories of graphs, Ann. Pure Applied Logic 53 (1991) 169-195.

25. D. Seese, Linear time computable problems and first-order descriptions, Math. Struct. in Computer Science (1996), in press.

26. L. Stockmeyer, The polynomial-time hierarchy, Theoret. Comput. Sci. 3 (1977) 1-22.

27. W. Thomas, Automata on infinite objects, in "Handbook of Theoretical Computer Science, vol. B", J. Van Leeuwen ed., Elsevier, 1990, pp. 133-192.

28. W. Tutte, Graph theory, Addison-Wesley, Reading, 1984.

29. I. Walukiewicz, Monadic second-order logic on tree-like structures, Proceedings STACS'96, Lec. Notes Comput. Sci., Vol. 1046, 1996, pp. 401-414.

LABRI, BordeAuX-1 University, 351 Cours de la Libération, 33405 TALENCE, France.

E-mail address: courcell@labri.u-bordeaux.fr.

WWW address: http://www.labri.u-bordeaux.fr/ courcell/courcell.html 